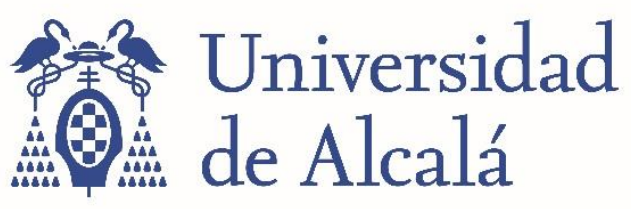

BIBLIOTECA

Document downloaded from the institutional repository of the University of Alcala: http://dspace.uah.es/

This is a postprint version of the following published document:

Gonzalez-Herraez, M.; Esteban, O.; Naranjo, F.B., 2007, "How to play with the spectral sensitivity of interferometers using slow light concepts and how to do it practically", Proc. SPIE 6619, 661937 (July 02, 2007)

Available at http://dx.doi.org/10.1117/12.738653

Copyright 2012 Society of Photo Optical Instrumentation Engineers. One print or electronic copy may be made for personal use only. Systematic electronic or print reproduction and distribution, duplication of any material in this paper for a fee or for commercial purposes, or modification of the content of the paper are prohibited.

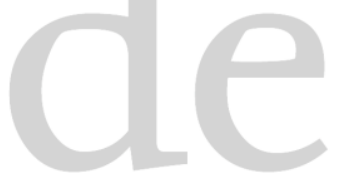

(Article begins on next page)

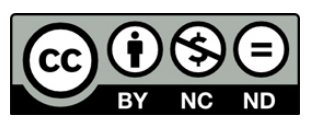

This work is licensed under a

Creative Commons Attribution-NonCommercial-NoDerivatives

4.0 International License. 


\title{
How to play with the spectral sensitivity of interferometers using slow light concepts - and how to do it practically
}

\author{
Miguel Gonzalez-Herraez, Oscar Esteban and Fernando B. Naranjo \\ Department of Electronics, Polytechnic School, University of Alcalá. \\ Alcalá de Henares, 28871 Madrid. Spain \\ Luc Thevenaz \\ Nanophotonics and Metrology Lab, Ecole Polytechnique Federale de Lausanne,
STI-NAM Section 11, Lausanne CH-1015, Switzerland
}

\begin{abstract}
We describe some interesting features that arise when slow and fast light structures are introduced in interferometers. With different configurations, one can obtain a spectral sensitivity enhancement of the interferometer or an enhanced robustness to laser frequency drifts. Furthermore, we describe simple practical ways to implement slow and fast light media in practical interferometers. We believe that these ideas may have interesting implications in optical sensing.
\end{abstract}

Keywords: slow light, interferometers, optical sensing, waveguides

\section{INTRODUCTION}

Slow light is known to be a very attractive approach to achieve all-optical delay lines and to provide a timing tool for photonic signal processing 1 . Successful experiments to control the light group velocity have been widely reported these past few years [2-8], showing the possibility to slow the speed of light up to nearly stopping it or to achieve group velocities exceeding the vacuum light velocity $c$. Most of these experiments use special media like cold atomic gases or electronic transitions in crystalline solids (such as ruby or alexandrite) working at well defined wavelengths. A significant step towards real applications has been achieved last year when slow light was experimentally and efficiently demonstrated in optical fibres using stimulated Brillouin scattering 9 (see also [10,11]) and later other nonlinear interactions with larger natural bandwidth but reduced efficiency 12 .

A slow light medium is a medium in which a very large group index $n_{g}$ is obtained. Conversely, a fast light medium is a medium in which a very small $(<1)$ or negative group index is obtained. The group index $\left(n_{g}\right)$ is related to the phase index $\left(n_{p}\right)$ by:

$$
n_{g}=n_{p}+\omega \frac{d n_{p}}{d \omega}
$$

The group index of the medium is related to the time delay $(\Delta t)$ of an optical pulse traveling through it by the following equation:

$$
\Delta t=\frac{n_{g} L}{c}
$$

All the experiments of slow and fast light performed up to now require the presence of narrowband spectral resonances in the medium. Spectral resonances have a complex response function, so that they introduce an extremely narrowband peak in the absorption/gain characteristics of the medium while there is also a sharp transition in the effective refractive index of the material. This sharp transition induces a strong change in the group index, which is responsible for large changes in the relative delay of an optical pulse as it travels through a material. This situation is depicted in Fig. 1, where the real and complex parts of the refractive index of a Lorentzian absorption have been depicted. 

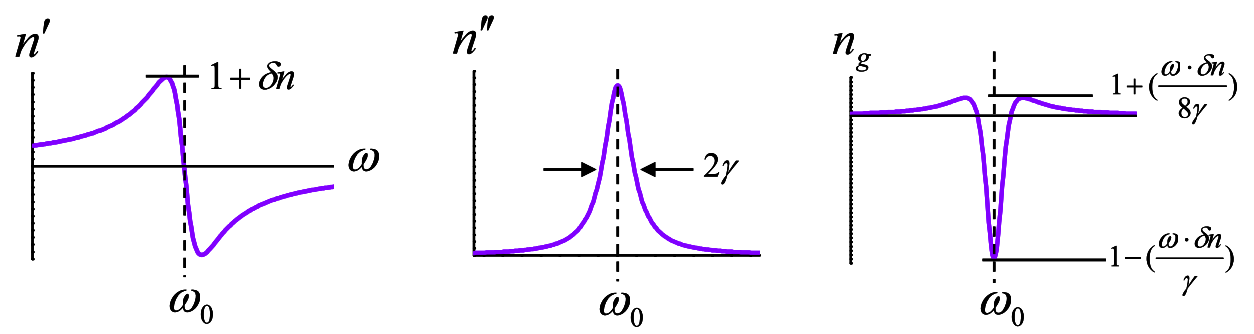

Fig 1.- Real (n') and imaginary (n") parts of the refractive index in a Lorentzian resonance, and the corresponding shape of the group index. A large decrease of the group index in the center of the resonance can be obtained.

It has been recently pointed out that slow light can have very interesting applications in optical sensing. Indeed, in the last years several proposals of optical sensors have been made that use slow and fast light concepts [15-19]. In particular, it is well-known that slow light may be used to enhance the sensitivity to electro-optic effects. The enhancement introduced by slow light in such devices is given by $n_{g}{ }^{2}$. This enhancement is caused by two effects: first, the effective interaction length in the medium is increased by a factor $n_{g}$; second, the optical intensity in the medium is also increased by the same amount $[15,16]$. Another potential application of slow light in sensing comes from the possibility of obtaining group velocities of light as small as the speed of sound. In this case, strong sound-matter interactions can be obtained with very interesting outcomes[17]. Some researchers have also pointed out the possibility of using slow light in interferometers [18] with large potential increases in the sensitivity. In this paper, we report on some interesting effects that can be obtained in interferometers when they include slow and fast light media. We observe that the spectral sensitivity of the interferometers can be largely enhanced (in the case of slow light) or reduced. Both effects are of great interest: in the case of an enhancement of the spectral sensitivity, this could be used to obtain impressive increases in the accuracy of frequency meters. In the case of a reduction of the sensitivity, this could be used to make the interferometer more robust to laser frequency drifts while preserving the sensitivity to length changes.

\section{SPECTRAL SENSITIVITY OF INTERFEROMETERS THAT USE SLOW AND FAST LIGHT MEDIA}

We start by considering a simple Fabry-Perot interferometer filled with a slow or fast light medium. Similar analysis apply to other types of interferometers, so this is just a particular case of a more general class of effects that are common to the use of slow light media in interferometers. In a Fabry-Perot interferometer, the transmission is given by:

$$
T(\delta)=\frac{(1-R)^{2}}{1+R^{2}-2 R \cos (\delta)}
$$

where $\mathrm{R}$ is the reflection coefficient of the facets and $\delta$ is the phase difference between two beams in a round-trip, hence:

$$
\delta=2 k L=2 \frac{\omega}{c} n_{p} L
$$

where we have assumed normal incidence. The transmission spectrum of a conventional Fabry-Perot exhibits resonances in frequency that are spaced by the free spectral range (FSR), being $F S R=c / n L$, and the width of these resonances is given by the finesse of the device $(F)$ through the relationship $\triangle f=F S R / F$. These relationships are obtained assuming that the refractive index of the medium inside the cavity does not change with frequency. In the presence of a strongly dispersive medium like a slow or fast light medium, these relationships change drastically. For simplicity, let us assume that we are centered at the peak of a Fabry-Perot resonance, hence $\delta=2 \pi m, m$ being an integer. A rapid look at equation (3) is enough to see that the width of the resonance is directly related to the rate of change of $\delta$ with frequency, hence:

$$
\frac{d \delta}{d \omega}=2 \frac{L}{c}\left(n_{p}+\omega \frac{d n_{p}}{d \omega}\right)=2 \frac{L}{c} n_{g}
$$


This is, the width of the resonance depends directly on the group index. Higher group indexes (slow light media) will yield narrower resonances, and smaller group indexes (fast light media) will yield broader resonances (potentially infinite for $\mathrm{n}_{\mathrm{g}}=0$, similar to a white light cavity [20]). In other words, with a slow light medium in the cavity, the spectral sensitivity of the device can be enhanced by a factor that is proportional to the group index. Taking into account that group indexes in the order of $10^{6}$ and $10^{7}$ have been demonstrated in atomic vapors, this sensitivity enhancement can be extremely important and can lead to unprecedented accuracy in some measurements.

Another surprising consequence is that, even if the spectral width of the device is changed, the sensitivity to length changes (displacements) remains absolutely intact. This is a very interesting and rather surprising outcome, and may be used to circumvent some usual trade-offs in these devices between FSR, finesse and spectral sensitivity. Again, we stress on the fact that these relationships apply for any kind of interferometer. For instance, in a Michelson interferometer the same number of fringes would be counted under the same length change, regardless of the medium used.

Of course, in all these derivations it should be taken into account that slow and fast light media are usually extremely narrowband, and hence these estimations can not hold over a wide wavelength range. But still, these rough estimations may indicate the great potentialities of slow light in interferometers.

\section{HOW TO MAKE PRACTICAL SLOW OR FAST LIGHT MEDIA}

Concerning slow and fast light media, there is one thing that has to be clear in the fiber sensing community: any medium exhibiting a sharp (gain or loss) resonance can potentially exhibit slow or fast light. Even though many of the experiments of slow and fast light have been performed in exotic media like atomic vapors, these are by no means necessary. We already showed in previous papers that slow and fast light can also be achieved in a conventional optical fiber (using stimulated Brillouin scattering) under the adequate pumping conditions [21].

In this section we prove that controllable fast light can be achieved in a simple, cheap and widely available optical material: in rhodamine $6 \mathrm{G}$ diluted in methanol. This medium exhibits a sharp absorption in the visible at a wavelength of $530 \mathrm{~nm}$, as shown in Fig. 2. The group index in the resonance can be simply tuned by increasing the rhodamine concentration in the solution.

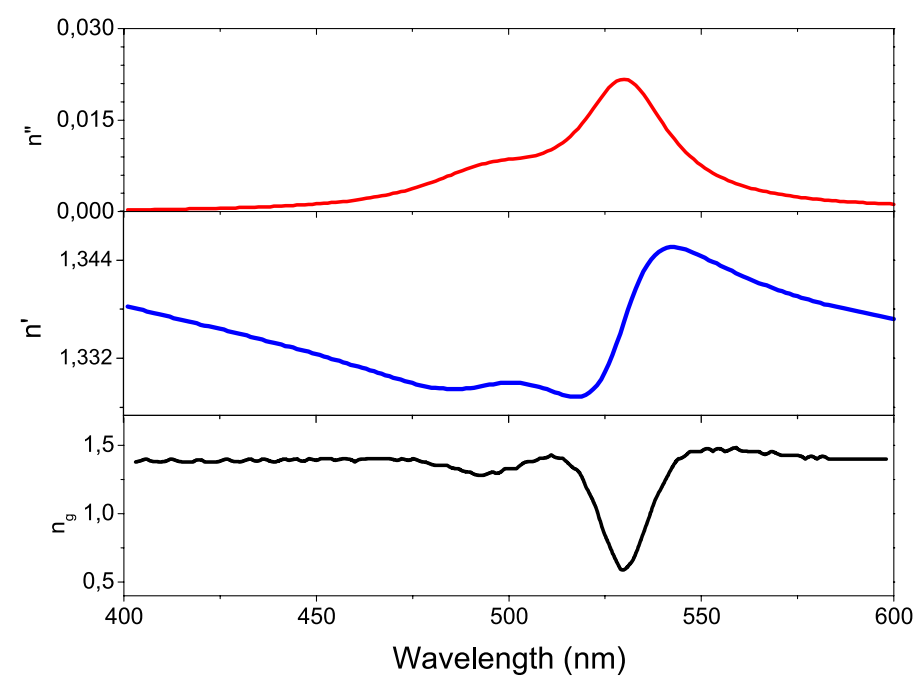

Fig 2.- Real (n') and imaginary (n") parts of the refractive index of rhodamine $6 \mathrm{G}$, diluted in methanol with a $0.02 \mathrm{M}$ concentration. The last graph shows the calculated group index for this material, which exhibits fast light at the wavelength of $530 \mathrm{~nm}$. With increasing concentration, one can obtain smaller values of the group index.

We believe that, under the adequate pumping conditions, this material can also exhibit slow light. However, pumping in this case may be costly. A simpler way to obtain slow light may come from making a mixture of two dyes with close 
resonances. In the spectral region between the resonances, the material is highly likely to exhibit slow light (a similar technique was used in [22] to create slow light in fiber from two loss resonances).

\section{CONCLUSIONS}

We have described some effects that arise when slow and fast light structures are introduced in interferometers. With different configurations, one can obtain a spectral sensitivity enhancement of the interferometer or an enhanced robustness to laser frequency drifts. Furthermore, we describe simple practical ways to implement slow and fast light media in practical interferometers by using dye solutions. We believe that these ideas may have interesting implications in optical sensing.

\section{Acknowledgments}

This work has been partially supported by Consejería de Educación de la Comunidad de Madrid research project FUTURSEN, ref. S0505/AMB-0374, by Fondo Social Europeo and Fondo Europeo de Desarrollo Regional, the Spanish Government research project HASSLE, ref. TEC2006-09990-C02-02, the Swiss National Science Foundation through project 200021-109773 and the COST Action 299.

\section{REFERENCES}

1. R. W. Boyd and D. J. Gauthier, in Progress in Optics, edited by E. Wolf, Vol. 43, Chap. 6, p.497.

2. A. M. Akulshin, S. Barreiro, and A. Lezema, Phys. Rev. Lett. 83, 4277 (1999).

3. M. D. Stenner, D. J. Gauthier, and M. A. Neifeld, Nature (London) 425, 695 (2003).

4. L. V. Hau, S. E. Harris, Z. Dutton, and C. H. Behroozi, Nature (London) 397, 594 (1999).

5. C. Liu, Z. Dutton, C. H. Behroozi, and L. V. Hau, Nature (London) 409, 490 (2001).

6. L. J. Wang, A. Kuzmich, and A. Dogariu, Nature (London) 406, 277 (2000).

7. M. S. Bigelow, N. N. Lepeshkin, and R. W. Boyd, Science 301, 200 (2003).

8. P. C. Ku, F. Sedgwick, C. J. Chang-Hasnain P. Palinginis, T. Li, H. L. Wang, S. W. Chang and S. L. Chuang, Opt. Lett. 292291 (2005).

9. K. Y. Song, M. G. Herráez, and L. Thévenaz, Opt. Express 13, 82-88 (2005).

10. Y. Okawachi, M.S. Bigelow, J. E. Sharping, Z. Zhu, A. Schweinsberg, D.J. Gauthier, R.W. Boyd, and A.L. Gaeta, Phys. Rev. Lett. 94153902 (2005).

11. J. T. Mok and B. J. Eggleton "Expect more delays" Nature 433811 (2005)

12. J. E. Sharping, Y. Okawachi, and A. L. Gaeta, Opt. Express 13, 6092 (2005).

13. D. Dahan and G. Eisenstein, Opt. Express 13, 6234 (2005)

14. J. E. Sharping, Y. Okawachi, J. van Howe, C. Xu, Y. Wang, A. E. Willner, and A. L. Gaeta, Opt. Express 13,7872 (2005).

15. M. M. Kash, V. A. Sautenkov, A. S. Zibrov, L. Hollberg, G. R. Welch, M. D. Lukin, Y. Rostovtsev, E. S. Fry and M. O. Scully, Phys. Rev. Lett. 825229 (1999)

16. M. Soljacic and J. D. Joannopoulos, Nature Materials 3, 211 (2004)

17. A. B. Matsko, Y. V. Rostovtsev, H. Z. Cummins and M. O. Scully, Phys. Rev. Lett. 845752 (2000).

18. Z. Shi, R. W. Boyd, D. J. Gauthier, C. C. Dudley Opt. Lett. (to be published)

19. M. Gonzalez-Herraez, Proc. OFS-18 (invited paper)

20. A. Wicht, K. Danzmann, M. Fleischhauer, M.O. Scully, G. Mueller, R.-H. Rinkleff Opt. Comm. 134 431 (1997)

21. M. Gonzalez-Herraez, K. Y. Song and L. Thevenaz Appl. Phys. Lett. 87081113 (2005)

22. K. Y. Song, M. Gonzalez-Herraez and L. Thevenaz Opt. Exp. 139758 (2005) 